\title{
Editorial: A Prospectus
}

With this inaugural issue, Survey of Digestive Diseases is launched. Our major objective is to present state-of-the-art reviews on clinical and basic science topics in gastrointestinal function and disorders. The articles will have as their goal the synthesis of current thinking and practice with the personal experience of the authors. For this purpose contributions will be drawn from a variety of disciplines - medicine, surgery, nutrition, radiology, pathology and the basic sciences. Although publication of original investigations is not the primary intent of the reviews, appropriate studies and series will be integrated into the presentation to provide a balanced view. A long-term objective is to bridge the gap between advances made in the academic setting, and their application to patient care. This can be achieved if, from the mass of medical literature, a judicious culling and understanding are rendered available to the clinician. The papers will not only consol-

idate but interpret the recent knowledge in the field of the digestive system.

The editorials in each issue will highlight a subject that appears timely and of general interest. Members of the Editorial Board will contribute their perspectives on current developments in their respective areas of special competence. From time to time an entire issue of the journal will be devoted to a 'symposium' on one topic. Other special and regular features will be introduced when deemed useful.

Survey of Digestive Diseases is conceived to serve the needs of clinicians, specialists and physicians-in-training. Their continual edification will contribute immeasurably to the maintenance of the high standard of medical practice. We hope the journal will provide the source of gastroenterologic information and wisdom for physicians everywhere.

Thomas S.N. Chen R.K. Zetterman 